\title{
Dependence Structure of Korean Financial Markets Using Copula-GARCH Model
}

\author{
Woohwan $\mathrm{Kim}^{1, a}$ \\ ${ }^{a}$ Financial Research \& Implementation, Korea
}

\begin{abstract}
This paper investigates the dependence structure of Korean financial markets (stock, foreign exchange (FX) rates and bond) using copula-GARCH and dynamic conditional correlation (DCC) models. We examine GJRGARCH with skewed elliptical distributions and four copulas (Gaussian, Student's $t$, Clayton and Gumbel) to model dependence among returns, and then employ DCC model to describe system-wide correlation dynamics. We analyze the daily returns of KOSPI, FX (WON/USD) and KRX bond index (Gross Price Index) from $2^{\text {nd }}$ May 2006 to $30^{\text {th }}$ June 2014 with 2,063 observations. Empirical result shows that there is significant asymmetry and fat-tail of individual return, and strong tail-dependence among returns, especially between KOSPI and FX returns, during the 2008 Global Financial Crisis period. Focused only on recent 30 months, we find that the correlation between stock and bond markets shows dramatic increase, and system-wide correlation wanders around zero, which possibly indicates market tranquility from a systemic perspective.
\end{abstract}

Keywords: Copula, asymmetric GARCH, tail dependence, dynamic conditional correlation model, system-wide correlation, financial crisis.

\section{Introduction}

The dependence structure of financial markets has been one of the most interesting issues in empirical finance, especially there is growing attention how to characterize it incorporating the empirical facts on observed returns, i.e. skewness, fat-tail and extreme comovement in tail-areas. We observe the simultaneous breakdown of markets during the 2008 Global Financial Crisis (GFC) and realize the magnitude of market malfunction is beyond our naive expectation. Under this circumstance, the investigation on dependence structure in systemic perspective has gained significant attraction. To properly model dependence among returns, it is essential to understand both individual specific characteristics and dependence between them. Unfortunately, it is too complicate to simply employ one of multivariate distributions. For instance, multivariate $t$ distribution indicates that each marginal should follow univariate $t$ distribution, however this is not well-supported for a empirical sense. Pesaran and Pesaran (2007) made a similar argument for a dynamic conditional correlation (DCC) structure.

During last decades, copula is regarded as one of the best solutions to model financial returns since it can model individual and joint separately. A copula is a function that joins or couples multivariate distribution and marginal distribution function. The basic idea of dependence modeling through copula is to enable the separation of the univariate margins and dependence structure, with the latter being completely described by copula, which makes it extremely useful to model a multivariate

\footnotetext{
This work was supported by the National Research Foundation of Korea Grant funded by the Korean Government [NRF2011-356-B00001].

${ }^{1}$ Financial Research \& Implementation (FRNI), 197-31 Donggyo-dong, Mapo-gu Seoul 121-896, Korea.

E-mail: jumnjump@gmail.com.
}

Published 30 September 2014 / journal homepage: http://csam.or.kr

(c) 2014 The Korean Statistical Society, and Korean International Statistical Society. All rights reserved. 
distribution in practice. In general, there are two copula classes; elliptical copula and Archimedean copula. The former is derived from the multivariate distribution function, including the Gaussian and Student's $t$ copulas, and the latter is derived using a generator, including Clayton, Frank and Gumbel copulas. Even though the popularity of elliptical copulas, they are criticized because of the symmetry and limitation for capturing extreme tail dependence, see Mashal and Zeevi (2002) and Dobric and Schmid (2005) and among others. Recently, Archimedean copulas are widely used to model asymmetric dependence among returns, see Joe (1997) and Nelsen (2006) for more detailed account on copula.

In origin, copula introduces to model dependence between i.i.d. data; consequently, it has a problem to apply return modeling due to the heteroskedasticity of observed returns. Previous studies introduce GARCH based (or GARCH filtered) copula models to incorporate heteroskedasticity. The copula-GARCH model combines GARCH and copula to enjoy flexibility in distributional assumption allowing heteroskedasticity and to overcome dimensional limitation of multivariate GARCH models. For detailed account on copula-GARCH model and its financial applications, see Jondeau and Rockinge (2006), Liu and Luger (2009), Huang et al. (2009) and Alouia et al. (2013). This paper employs copula-GARCH model to investigate the dependence structure of Korean financial markets, stock, foreign exchange (FX) rates and bond. We employ standard GARCH of Bollerslev (1986) and GJR-GARCH of Glosten et al. (1993) with skewed elliptical distributions for marginal modeling, and then apply four copulas, Gaussian, $t$, Clayton and Gumbel copulas, to model dependence among returns. The static approach is also restricted to examine the dependence structure of empirical returns because of its time varying property; consequently, we use DCC to describe correlation dynamics over times.

We analyze the daily returns of stock (KOSPI), FX rates (WON/USD) and bond (KRX Gross Price Index), ranged from $2^{\text {nd }}$ May 2006 to $30^{\text {th }}$ June 2014 with 2,063 observations. To better understand the impact of GFC to Korean markets, we construct two sub-samples, denoted as GFC and Recent in the following. The former is covered from $2^{\text {nd }}$ January 2008 to $30^{\text {th }}$ December 2009, and the latter is from $2^{\text {nd }}$ January 2012 to $30^{\text {th }}$ June 2014. On marginal distribution, we find that GJR-GARCH with skew- $t$ distribution is a good candidate during GFC, whereas GJR-GARCH with normal distribution shows good in-sample fit in recent period, which indicates that there is strong asymmetric effect in Korean markets. In terms of dependence, our results suggest that there is strong dependence between KOSPI and FX returns during GFC, whereas, the correlation between stock and bond markets shows dramatic increase in recent period. We find that the best copula to describe the dependence in Korean markets is $t$ copula, and the estimated shape parameter is 13 in GFC period, which implies the strong tail dependence among returns, and may lead to the simultaneous break-downs of Korean financial markets. It is noticeable that the static correlations between pair-wise returns are negligible with a negative magnitude; however, dynamic correlations show dramatic movement both up and downward according to market changes. We note that the parameter estimates of Clayton and Gumbel copulas are unable to obtain because of negative correlation (also Kendall's tau). Finally, the system-wide dynamic correlation wanders around zero during last two years, which is possibly related to market tranquility in systemic perspective.

The rest of this paper is organized as follows. Section 2 briefly provides how to model each individual return using GJR-GARCH model and reviews copula functions. Section 3 presents empirical results and discusses our findings. Section 4 concludes. Appendix 1 reviews the DCC model of Engle (2002). 


\section{Copula-GARCH Models}

\subsection{Marginal modeling}

Let $r_{t}=\left(r_{1, t}, \ldots, r_{d, t}\right)$ be a $d$-dimensional vector of asset returns at time $t$. To deal with both the asymmetric shape and heteroskedasticity of observed return, we consider univariate GJR-GARCH $(1,1)$ model with skew elliptical distributions to model each individual return. Formally, individual return $r_{i, t}$, for $i=1, \ldots, d$, is expressed as

$$
\begin{aligned}
r_{i, t} & =\mu_{i, t}+\varepsilon_{i, t}=\mu_{i, t}+\sigma_{i, t} z_{i, t}, \\
\sigma_{i, t}^{2} & =\alpha_{i, 0}+\alpha_{i, 1} \varepsilon_{i, t-1}^{2}+\alpha_{i, 2} \sigma_{i, t-1}^{2}+\alpha_{i, 3} 1_{\left\{\varepsilon_{i, t-1}<0\right\}} \varepsilon_{i, t-1}^{2}
\end{aligned}
$$

where $\mu_{i}$ denotes the conditional mean based on the information up to $t-1$, which is modeled as either a constant $\left(\mu_{i}=\mu\right)$ or the $p$-th order autoregressive model $\left(\mu_{i}=\mu_{i}+\sum_{l=1}^{p} \gamma_{i} r_{t-i}\right)$ depending on the characteristics of each return. The innovation $z_{i, t}$ has zero mean and unit variance, and follows one of skewed elliptical distributions. This paper considers four candidates to model innovations: normal, Student's $t$, skew-normal and skew-t distributions. The $1_{\{\}}$is an usual indicator function which takes on value 1 if condition holds, and 0 otherwise. The four parameters of conditional variance given in (2.2), $\alpha_{i, 0}, \alpha_{i, 1}, \alpha_{i, 2}$ and $\alpha_{i, 3}$, are positive and holds $\alpha_{i, 1}+\alpha_{i, 2}+1 / 2 \alpha_{i, 3}<1$. We note that a leverage effect is captured by the GJR-GARCH model when $\alpha_{i, 3}>0$, and GJR-GARCH reduces to standard GARCH when $\alpha_{i, 3}=0$. For notational convenience, all parameters of each marginal distribution are collected in $\phi_{i}=\left\{\mu_{i, t}, \alpha_{i, 0}, \alpha_{i, 1}, \alpha_{i, 2}, \alpha_{i, 3}, v_{i}, \delta_{i}\right\}$, for $i=1, \ldots, d$, where $v_{i}$ is the shape parameter of $t$ or skew- $t$ distribution and $\delta_{i}$ denotes the skew parameter of skew-normal or skew- $t$ distribution.

\subsection{Copula}

By applying copula theory, a joint distribution of $d$-dimensional vector is expressed as

$$
F\left(r_{1, t}, \ldots, r_{d, t} ; \theta\right)=C\left(F_{1}\left(r_{1} ; \phi_{1}\right), \ldots, F_{d}\left(r_{d} ; \phi_{d}\right) ; \theta\right),
$$

where $C$ is a $d$-dimensional copula function with parameter $\theta$ and $F_{i}\left(r_{i} ; \phi_{i}\right)$ is the $i$ th marginal distribution function parameterized by $\phi_{i}$, for $i=1, \ldots, d$.

The conditional density of elliptical copulas at time $t$ is given by

$$
c\left(u_{1, t}, \ldots, u_{d, t}\right)=\frac{f\left(F_{1}^{-1}\left(u_{1, t} ; \phi_{1}\right), \ldots, F_{d}^{-1}\left(u_{d, t} ; \phi_{d}\right)\right)}{\prod_{l=1}^{d} f_{i}\left(F_{i}^{-1}\left(u_{i, t} ; \phi_{i}\right) ; \theta\right)},
$$

where $u_{i}=F_{i}\left(r_{i, t} ; \phi_{i}\right)$ is the probability integral transformation (PIT) of each series by its conditional distribution $F_{i}$ estimated via the first stage GARCH process, $f(; \theta)$ is the multivariate density of either multivariate normal or Student's $t$ distribution with parameter vector $\theta$.

Table 1 provides the functional form of Gaussian, Student's $t$, Clayton and Gumbel copulas. The parameter of Gaussian copula is linear correlation matrix $\Sigma$ which characterizes the dependence of random variables. The tail dependence of the Gaussian copula is zero, except for only perfect correlation. The parameters of Student's $t$ copula are linear correlation matrix and degree of freedom $(d f, v)$, the latter characterizes the co-movement in tail area among random variables. The lower $d f$ implies the strong co-movement in the tail area. The tail dependence coefficient of the Student's $t$ copula is given by $\lambda_{U}=\lambda_{L}=2 t_{v+1}(-\sqrt{v+1} \sqrt{(1-\rho) /(1+\rho)})$, where $\rho$ is pairwise correlation coefficient. We note that Student's $t$ copula is a suitable candidate to reflect symmetric tail dependence. The parameter of Gumbel copula controls the strength of dependence; when $\theta=1$, there is no dependence 
Table 1: Copula function

\begin{tabular}{ccc}
\hline \hline Copula & Functional Form & Parameter Space \\
\hline Gaussian & $C\left(u_{1}, \ldots, u_{d} ; \Sigma\right)=\int \cdots \int \frac{1}{(2 \pi)^{d / 2}|\Sigma|^{1 / 2}} \exp \left(-\frac{1}{2} u^{\prime} \Sigma^{-1} u\right) d u$ & \\
Student's $t$ & $C\left(u_{1}, \ldots, u_{d} ; \Sigma, v\right)=\int \cdots \int \frac{\Gamma(v+d / 2)}{\Gamma(v / 2)(v \pi)^{d / 2}|\Sigma|^{1 / 2}}\left(1+\frac{1}{v} u^{\prime} \Sigma^{-1} u\right)^{-(v+d) / 2} d u$ & $0<v<\infty$ \\
Clayton & $C\left(u_{1}, \ldots, u_{d} ; \theta\right)=\left(\sum_{i=1}^{d} u_{i}^{-\theta}-1\right)^{-1 / \theta}$ & $0<\theta<\infty$ \\
Gumbel & $C\left(u_{1}, \ldots, u_{d} ; \theta\right)=\exp \left\{-\left(-\sum_{i=1}^{d} \ln u_{i}^{\theta}\right)^{1 / \theta}\right\}$ & $\theta \geq 1$ \\
\hline \hline
\end{tabular}

Note: The integrations in Gaussian and $t$ copulas are component-wise integration and $|\cdot|$ denotes matrix determinant.

and when $\theta \rightarrow \infty$, there is perfect dependence. Gumbel copula exhibits only upper tail dependence, $\lambda_{U}=2-2^{1 / \theta}$, whereas Clayton copula shows only lower tail dependence, $\lambda_{L}=2^{-1 / \theta}$.

For practical application, we need to estimate copula parameters and select a best copula based on given data. This paper applies two-stage approach to obtain the parameter estimates in copulaGARCH models. We decide each marginal distribution using GARCH models and then apply PIT to each return, in the first stage. The copula parameter is obtained in the second step using PIT observation in the first step. Under the standard regularity condition, it is well-known that two-stage method yields the consistent and asymptotically normal estimator. The copula selection is based on Bayesian information criterion (BIC). For more details on parameter estimation and copula selection, see Joe (1997) and Nelsen (2006).

\section{Empirical Analysis}

\subsection{Data}

The empirical data consists of the daily returns of stock index (KOSPI), FX rates (WON/USD) and bond index (KRX Bond Gross Price index, hereafter KRX Bond), covered from $2^{\text {nd }}$ May 2006 to $30^{\text {th }}$ June 2014, totally 2,063 observations. The KOSPI and KRX bond indices are issued by Korea Stock Exchange and obtained from http://www.krx.co.kr, and FX data is available from Bank of Korea via http://ecos.bok.or.kr. To better understand the impact of GFC to Korean markets, we construct two sub-samples, named as GFC and Recent. The GFC is ranged from $2^{\text {nd }}$ January 2008 to $30^{\text {th }}$ December 2009 (500 observations) and Recent is from $2^{\text {nd }}$ January 2012 to $30^{\text {th }}$ June 2014 (616 observations). The continuous compound rate of return is calculated as $r_{t}=100 \times \ln \left(p_{t} / p_{t-1}\right)$, where $p_{t}$ denotes the daily closing price at time $t$.

Figure 1 depicts the time series plots of KOSPI, FX and KRX Bond at level. As many studies point out, we observe the simultaneous break-down during GFC, surprising downside spike of KOSPI and upside jump of FX rates. The KOSPI shows a clear increasing pattern during the first two years (2006 2007), and then it sharply decreases from January 2008 to May 2009, closely caused by GFC. As GFC gone through, KOSPI has another increasing momentum lasting for two years (May 2009 May 2011). In May of 2011, there is a short-live downside spike, and then it wanders around 1950 level after 2012. Secondly, the dynamics of FX level show a stable movement for the first two years, and then there is a dramatic increase starting from May 2008, and FX rates reaches its maximum level at the end of 2008. After May of 2009, FX shows gradual decreasing and stabilizes around 1000. The KRX Bond also shows a quite volatile movement during GFC and interestingly we observe the 


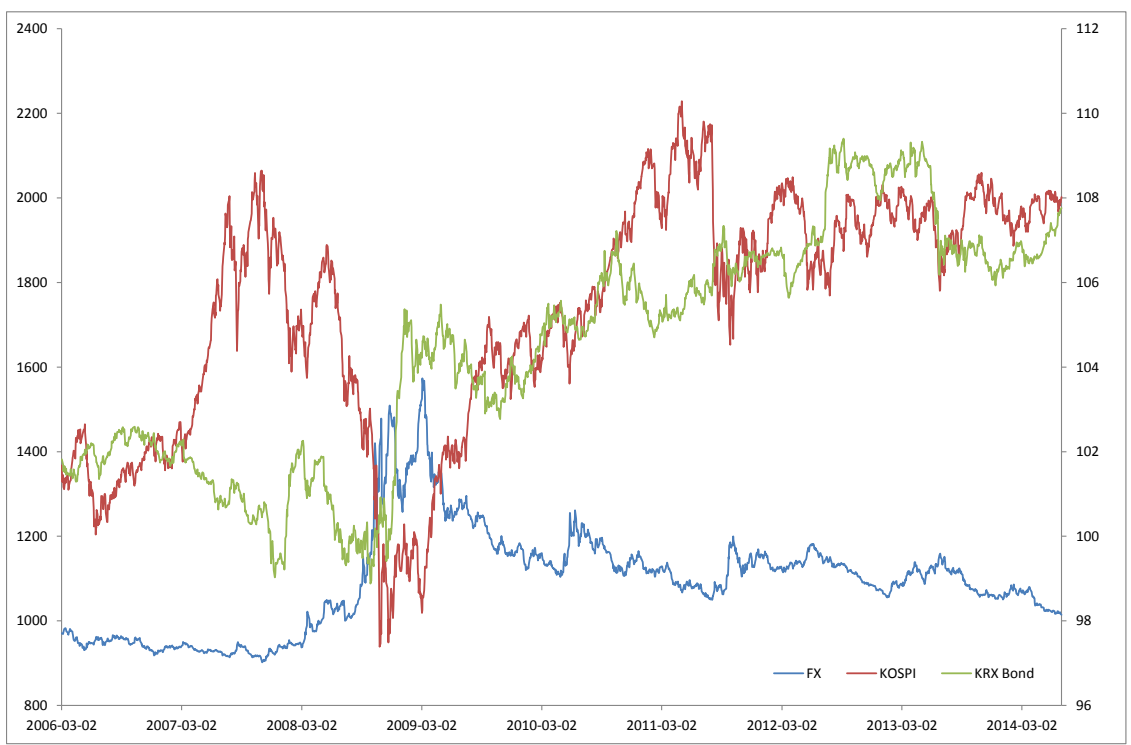

Figure 1: Time plots of KOSPI, FX and KRX bond index

Note: Daily prices(levels) of the KOSPI, FX and KRX bond index from $2^{\text {nd }}$ March 2006 to $30^{\text {th }}$ June 2014.

Table 2: Summary statistics

\begin{tabular}{|c|c|c|c|c|c|c|}
\hline & \multicolumn{2}{|c|}{ KOSPI } & \multicolumn{2}{|c|}{ FX } & \multicolumn{2}{|c|}{ KRX Bond } \\
\hline & $\overline{\text { GFC }}$ & Recent & GFC & Recent & $\overline{\mathrm{GFC}}$ & Recent \\
\hline Min & -11.172 & -3.4615 & -9.195 & -1.2281 & -0.9671 & -0.5444 \\
\hline Max & 11.2844 & 2.8875 & 7.14 & 1.4249 & 0.7873 & 0.724 \\
\hline Mean & -0.0177 & 0.015 & 0.0437 & -0.0214 & 0.0077 & 0.0017 \\
\hline SD & 2.0614 & 0.8398 & 1.309 & 0.3573 & 0.1962 & 0.112 \\
\hline Skewness & -0.4628 & -0.0125 & -0.7508 & 0.2801 & -0.3436 & -0.4727 \\
\hline Kurtosis & 5.3275 & 1.3148 & 12.6462 & 0.6226 & 2.8239 & 6.574 \\
\hline $\mathrm{JB}$ & $616.7^{* * *}$ & $45.34^{* * *}$ & $3412^{* * *}$ & $18.42^{* * *}$ & $178.8^{* * *}$ & $1143^{* * *}$ \\
\hline $\mathrm{LB}(10)$ & 4.214 & 11.61 & $54.36^{* * *}$ & $22.46^{* *}$ & 8.302 & 8.243 \\
\hline $\mathrm{LB}^{2}(10)$ & $326.6^{* * *}$ & $73.57^{* * *}$ & $228.9^{* *}$ & $66.01^{* * *}$ & 14.43 & $38.98^{* * *}$ \\
\hline
\end{tabular}

Note: SD denotes standard deviation. JB and LB(10) stand for the Jarque-Bera and Ljung-Box Q statistic with order=10, respectively. The former is for normality test and the latter is for autocorrelation test. The $\mathrm{LB}^{2}$ is Ljung-Box statistic using squared returns to examine autocorrelation in second order sense. The ${ }^{* * *}$ and ${ }^{* *}$ denote the statistically significant at $1 \%$ and $5 \%$ significance level, respectively. The number of observations in GFC period ( $2^{\text {nd }}$ January $2008 \sim 30^{\text {th }}$ December 2009)is 500, that of the Recent period ( $2^{\text {nd }}$ January $2012 \sim 30^{\text {th }}$ June 2014) is 616.

structural shift at level after GFC. The KRX Bond shows gradual increasing movement after May of 2009.

Table 2 provides the summary statistics of three returns. The KOSPI and FX returns show dramatic changes caused by financial crisis, simply based on the fact that the mean returns of both KOSPI and FX are changed from negative to positive, and the standard deviation of each return increases over three times compared to recent period. The minimum returns of KOSPI, FX and KRX Bond are realized at $24^{\text {th }}$ October 2008, $14^{\text {th }}$ October 2008 and at $10^{\text {th }}$ September 2009, respectively. Based on this result, we may say that the market responses for external shock, i.e. GFC show somewhat similar, regardless of market specific characteristics, especially when the magnitude of the shock is extreme 
Table 3: Static correlation coefficients

\begin{tabular}{clcc}
\hline \hline & KOSPI & FX & KRX Bond \\
\cline { 2 - 4 } & & GFC & \\
\hline KOSPI & 1 & 1 & 1 \\
FX & $-0.1760^{* * *}$ & 0.0153 & \\
KRX Bond & -0.0155 & Recent & 1 \\
\hline KOSPI & 1 & 1 & \\
FX & -0.0212 & -0.0201 & 1 \\
KRX Bond & $-0.1473^{* * *}$ & & \\
\hline \hline
\end{tabular}

Note: The ${ }^{* * *}$ denotes the statistically significant at $1 \%$ significance level.

Table 4: BIC of various GARCH models

\begin{tabular}{|c|c|c|c|c|c|c|}
\hline & \multicolumn{2}{|c|}{ KOSPI } & \multicolumn{2}{|c|}{ FX } & \multicolumn{2}{|c|}{ KRX Bond } \\
\hline & GFC & Recent & GFC & Recent & GFC & Recent \\
\hline GARCH with Normal & 3.975 & 2.451 & 2.597 & 0.7045 & -0.4326 & -1.599 \\
\hline GARCH with $t$ & 3.953 & 2.446 & 2.542 & 0.7113 & -0.5554 & -1.859 \\
\hline GARCH with skew-normal & 3.966 & 2.458 & 2.602 & 0.7140 & -0.4470 & -1.596 \\
\hline GARCH with skew- $t$ & 3.950 & 2.454 & 2.548 & 0.7214 & -0.5559 & -1.860 \\
\hline GJR-GARCH with Normal & 3.949 & 2.427 & 2.602 & 0.6677 & -0.4641 & -1.596 \\
\hline GJR-GARCH with $t$ & 3.933 & 2.429 & 2.548 & 0.6766 & -0.5560 & -1.849 \\
\hline GARCH with skew-normal & 3.935 & 2.435 & 2.610 & 0.6782 & -0.4764 & -1.586 \\
\hline GJR-GARCH with skew- $t$ & 3.926 & 2.437 & 2.555 & 0.6871 & -0.5596 & -1.850 \\
\hline
\end{tabular}

large. The standard deviation of stock return shows the highest level, more specifically almost double than that of FX, and eight times higher than the bond index, which implies the stock market is the most risky market in Korea. We can see negative skewness and strong excess kurtosis, commonly found in many financial return. The Jarque-Bera (JB) normality test confirms the non-normality of each return series at the $1 \%$ significance level. In addition, we test the presence of autocorrelation in return and squared return using Ljung-Box statistics, denoted by $\mathrm{LB}$ and $\mathrm{LB}^{2}$ in Table 2 . The null hypotheses of no autocorrelation are rejected in all cases of squared return series at the $1 \%$ significance level, which indicates the existence of strong ARCH effect.

Table 3 provides the static pair-wise correlations between returns. We see the weak correlation between all pairs, whose average degree are -0.17 in GFC and -0.15 in recent periods. Even the average degrees are similar both periods, whereas the specific characteristics are somewhat different. The pair with significant correlation is KOSPI-FX in GFC, and that of recent period is KOSPI-KRX Bond.

\subsection{Marginal Results}

Before applying GRACH model, we analyze the conditional mean for each return. Based on the significance test and BIC, we conclude that the conditional mean of KOSPI and KRX Bond are constant, whereas that of FX return is modeled using AR(4). Our results coincide with previous studies; constant mean of stock return and strong autoregressive structure of FX return. Table 5 indicates the estimation results of the conditional mean.

To decide the best model for describing heteroskedasticity, we mainly examine GARCH and GJRGARCH with normal, $t$, skew-normal and skew- $t$ distributions. The following results are obtained using $\mathrm{R}$ package rugarch. The BIC values of various GARCH models are presented in Table 4, and the best candidate for each return is highlighted with bold style. Generally speaking, the GJR-GARCH with skew-t model and GJR-GRACH with normal distribution models conclude as suitable candidates 
Table 5: Parameter estimates of GARCH models

\begin{tabular}{|c|c|c|c|c|c|c|}
\hline & \multicolumn{2}{|c|}{ KOSPI } & \multicolumn{2}{|c|}{ FX } & \multicolumn{2}{|c|}{ KRX Bond } \\
\hline & GFC & Recent & GFC & Recent & GFC & Recent \\
\hline$\mu$ & -0.0177 & 0.0150 & $0.0375^{*}$ & -0.0189 & 0.0077 & 0.0017 \\
\hline$\gamma_{1}$ & NA & NA & $0.2744^{* * *}$ & $0.1067^{* * *}$ & NA & NA \\
\hline$\gamma_{2}$ & NA & NA & $-0.0782^{* * *}$ & $-0.0217^{* * *}$ & NA & NA \\
\hline$\gamma_{3}$ & NA & NA & $-0.1628^{* * *}$ & $0.0351^{* * *}$ & NA & NA \\
\hline$\gamma_{4}$ & NA & NA & $0.0993^{* * *}$ & $-0.0549^{* * *}$ & NA & NA \\
\hline$\alpha_{0}$ & $0.0625^{* *}$ & $0.0123^{*}$ & 0.0262 & $0.0016^{* *}$ & $0.0008^{* *}$ & 0.0005 \\
\hline$\alpha_{1}$ & 0.0000 & 0.0000 & 0.2570 & $0.0727^{* * *}$ & $0.0898^{* * *}$ & $0.1101^{*}$ \\
\hline$\alpha_{2}$ & $0.9034^{* * *}$ & $0.9403^{* * *}$ & 0.7420 & $0.9663^{* * *}$ & $0.9408^{* * *}$ & $0.8801^{* * *}$ \\
\hline$\alpha_{3}$ & $0.1483^{* * *}$ & $0.0789^{* * *}$ & NA & $-0.1085^{* * *}$ & $-0.0891^{* *}$ & NA \\
\hline$\delta$ & $0.8418^{* * *}$ & NA & $5.1760^{* * *}$ & NA & $0.8573^{* * *}$ & $3.1510^{* * *}$ \\
\hline$v$ & $7.6200^{* * *}$ & NA & NA & NA & $4.5105^{* * *}$ & NA \\
\hline
\end{tabular}

during GFC and recent periods, respectively.

Table 5 presents the parameter estimates of GARCH models. As previous studies point out, we find a strong persistence in time-varying volatility dynamics, since $\alpha_{1}+\alpha_{2}$ in GARCH and $\alpha_{1}+$ $\alpha_{2}+1 / 2 \alpha_{3}$ in GJR-GARCH are close to unity. The integrated GARCH (IGARCH) and fractionally integrated GARCH (FIGARCH) are possible candidates to reflect strong persistence. Previous studies such as Mikosch and Starica (2004) and Hillebrand (2005) argued that the estimated persistence in various GARCH models is known to be too large when the parameters of the model undergo structural changes somewhere in the sample. Unfortunately, it is difficult task to find why this strong persistence is caused by the data, the parameter change or external shocks, and our primary purpose of GARCH model is to obtain the i.i.d. residual for further copula modeling. Thus, we do not pay much attention to this issue. We find that there is significant asymmetric effect in stock return, since the KOSPI is positive with statistical significance both GFC and recent periods. Similar to previous findings, the estimates of shape parameter indicate a fat-tailed and asymmetric shape when t or skew- $t$ distributions are used, i.e. KOSPI, FX and KRX Bond returns in GFC period. We note that the best candidate for the conditional distribution of GARCH model in KOSPI and FX returns is normal distribution in the recent period, which may indicate market tranquility during recent years.

Figure 2 deficits the time-varying volatility of each return using best GARCH models. As we mentioned based on standard deviation, the volatility of KOSPI is higher than other returns. The volatilities of KOPSI and FX returns reach their highest level at the end of 2008, due to GFC impact. We note that the absolute magnitude of each volatility Recent period significantly reduces, compared to that of GFC period.

\subsection{Copula results}

To examine pairwise dependence, we fit bivariate Gaussian, Student's $t$, Clayton and Gumbel copulas. Table 6 provides the results. We only provide the parameter estimates of Gaussian and Student's $t$ copulas since those of Clayton and Gumbel copulas are not obtainable in some cases, which will be discussed in the following sub-section. For the time being, we focus on Gaussian and Student's $t$ copulas. Based on BIC values, it seems difficult to reach a general conclusion on the dependence of each pair. At first, the copula for KOSPI-FX is Gaussian for both periods, and the KOSPI-KRX Bond is changed from Gaussian to $t$ copula, whereas the FX-KRX Bond is exactly contrary to KOSPIKRX Bond. As many studies indicate, we find that the dependence between returns shows strong tail-dependence which advocates the use of $t$ copula. We will discuss the reason why our results are 


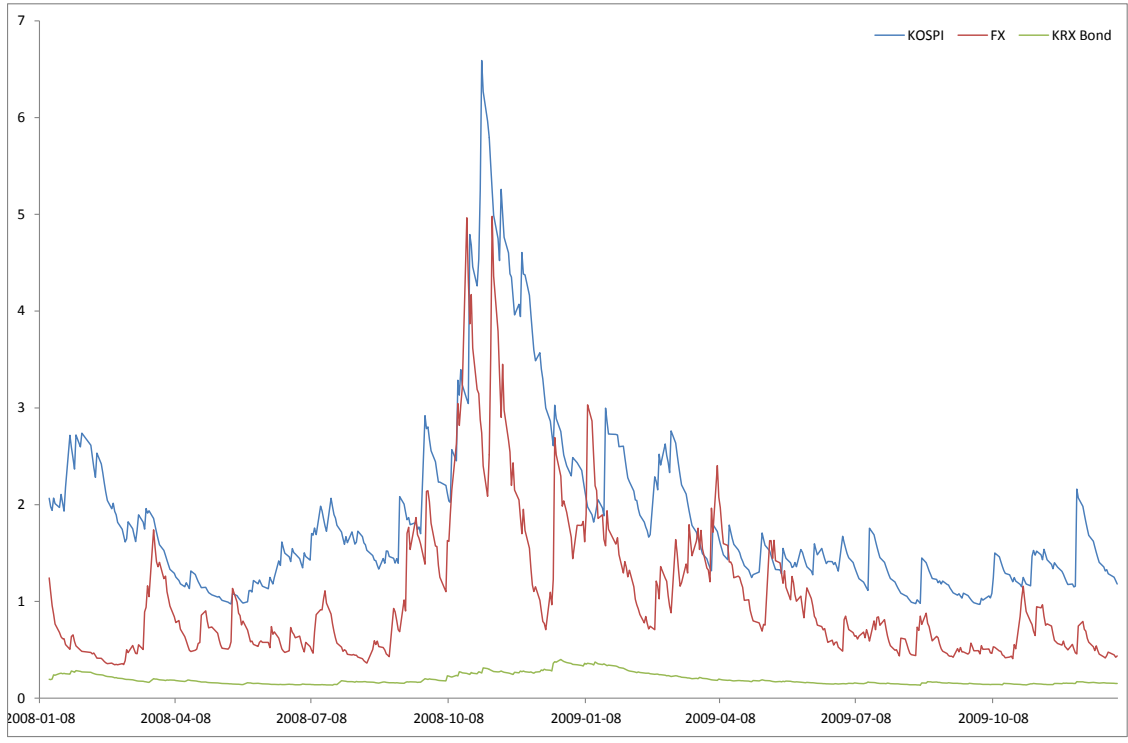

(a) GFC

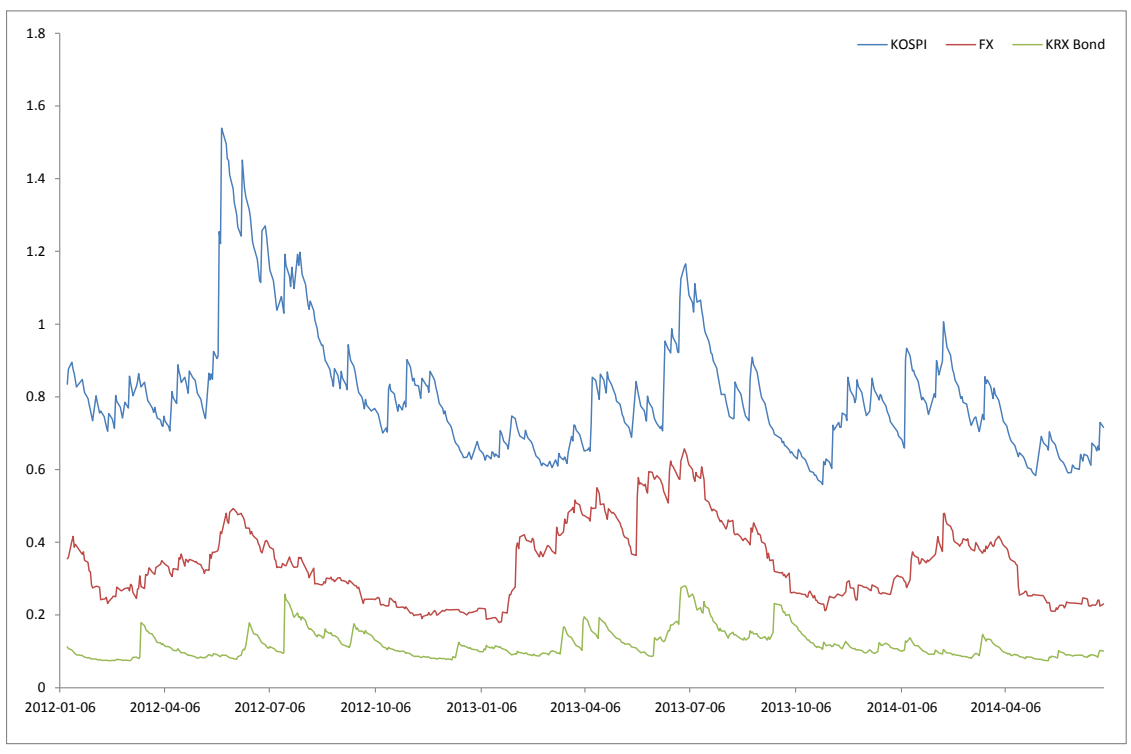

(b) Recent

Figure 2: Volatility dynamics of KOSPI, FX and KRX bond index

Note: Table 4 shows the conditional volatility is obtained from the best GARCH models. The $y$-axis represents the daily volatility in $\%$.

complicated in the following sub-section.

Next, we try to fit of three-dimensional Gaussian, Student's $t$, Clayton and Gumbel copulas. The 
Table 6: Two-dimensional copula estimation results

\begin{tabular}{cccc}
\hline \hline Pair & Period & Gaussian copula & Student's $t$ copula \\
\hline \multirow{2}{*}{ KOSPI-FX } & GFC & -0.0578 & $\rho=-0.0579, v \rightarrow \infty$ \\
& & $\mathbf{9 . 8 1 6 9}$ & 21.2338 \\
\cline { 2 - 4 } & \multirow{2}{*}{ Recent } & -0.0048 & $-0.0047, v \rightarrow \infty$ \\
& & $\mathbf{1 1 . 3 9 3 3}$ & 22.8109 \\
\hline \multirow{2}{*}{ KOSPI-KRX Bond } & GFC & $-0.1163^{* * *}$ & $\rho=-0.1385^{* * *}, v=6.5835$ \\
& & $\mathbf{4 . 9 4 6 9}$ & 8.7738 \\
\cline { 2 - 4 } & \multirow{2}{*}{ Recent } & $-0.2139^{* * *}$ & $\rho=-0.2158, v=4.9824$ \\
& & -16.3931 & $-\mathbf{2 2 . 3 8 6 2}$ \\
\hline \multirow{2}{*}{ FX- KRX Bond } & \multirow{2}{*}{ GFC } & 0.0304 & $\rho=0.0238^{* * *}, v=17.6726$ \\
& & 10.9669 & $\mathbf{8 . 7 7 3 8}$ \\
\cline { 2 - 4 } & \multirow{2}{*}{ Recent } & -0.0640 & $\rho=-0.0067, v \rightarrow \infty$ \\
& & $\mathbf{1 1 . 3 8 2 3}$ & 22.8109 \\
\hline \hline
\end{tabular}

Note: The ${ }^{* * *}$ and ${ }^{* *}$ denote the statistically significant at $1 \%$ and 5\% significance levels, respectively. The parameter of Gaussian copula is linear correlation coefficient, denoted by $\rho$. The Student's $t$ copula is two-parameter copula; linear correlation $(\rho)$ and shape parameter $(v)$, known as degree of freedom.

Table 7: Three-dimensional copula estimation results

\begin{tabular}{ccrr}
\hline \hline & $\theta$ & BIC \\
\cline { 2 - 4 } & $\rho_{12}=-0.0579, \rho_{13}=-0.1163^{* * *}, \rho_{23}=0.0306$ & 25.8808 \\
GFC & $\rho_{12}=-0.0048, \rho_{13}=-0.2139^{* * *}, \rho_{23}=-0.0064$ & 6.4208 \\
Recent & Student's $t$ copula & $\mathbf{2 0 . 7 8 0 8}$ \\
\hline GFC & $\rho_{12}=-0.0524, \rho_{13}=-0.1273^{* * *}, \rho_{23}=0.0283, v=13.74$ & $\mathbf{3 . 2 2 0 8}$ \\
Recent & $\rho_{12}=-0.0009, \rho_{13}=-0.2208^{* * *}, \rho_{23}=-0.0134, v=20.34$ & Gaussian copula \\
\hline \hline
\end{tabular}

Note: The ${ }^{* * *}$ and ${ }^{* *}$ denote the statistically significant at $1 \%$ and $5 \%$ significance levels, respectively. The parameter of Gaussian copula is linear correlation coefficient, denoted by $\rho_{i j}$, for $i, j=1,2,3$. The Student's $t$ copula is two-parameter copula; linear correlation $\left(\rho_{i j}\right)$ and a shape parameter $(v)$.

parameter estimates of Gaussian and Student's $t$ copulas are presented in Table 7, and we also note that those of Clayton and Gumbel copulas are not reported because of the similar reason in twodimensional case. The three-dimensional results coincide with our prior knowledge based on previous studies. The best copula is $t$ copula in both periods, furthermore the shape parameter of $t$ copula (denoted by $v$ in Table 7) is below 20. Especially, estimated shape parameter $v$ of $t$ copula in GFC is 13, which indicates the strong tail dependence among returns during GFC.

\subsection{Correlation dynamics}

To improve our understanding of dependence structure over time and find out why the parameter estimates of Clayton and Gumbel copulas are unable to obtain in previous sub-section, we conduct rolling window analysis and exponentially weighted moving average (EWMA) correlation. We use 252-day (approximately one year) moving windows rolling analysis based on Pearson's correlation coefficient. The estimator of EWMA correlation at time is given by

$$
\hat{\rho}_{i j, t}^{\mathrm{EWMA}}=\frac{(1-\lambda) \sum_{l=0}^{k-1} \lambda^{l}\left(r_{i, k-l}-\bar{r}_{i}\right)\left(r_{j, k-l}-\bar{r}_{j}\right)}{\sqrt{(1-\lambda) \sum_{l=0}^{k-1} \lambda^{l}\left(r_{i, k-l}-\bar{r}_{i}\right)^{2}} \sqrt{(1-\lambda) \sum_{l=0}^{k-1} \lambda^{l}\left(r_{j, k-l}-\bar{r}_{j}\right)^{2}}},
$$

where $\bar{r}_{i}$ is sample mean and the decay (or smoothing) parameter $\lambda$ determines the relative weight assigned to each observation. RiskMetrics (1996) recommends $\lambda=0.97$ for daily data $(\lambda=0.94$ for monthly data). 


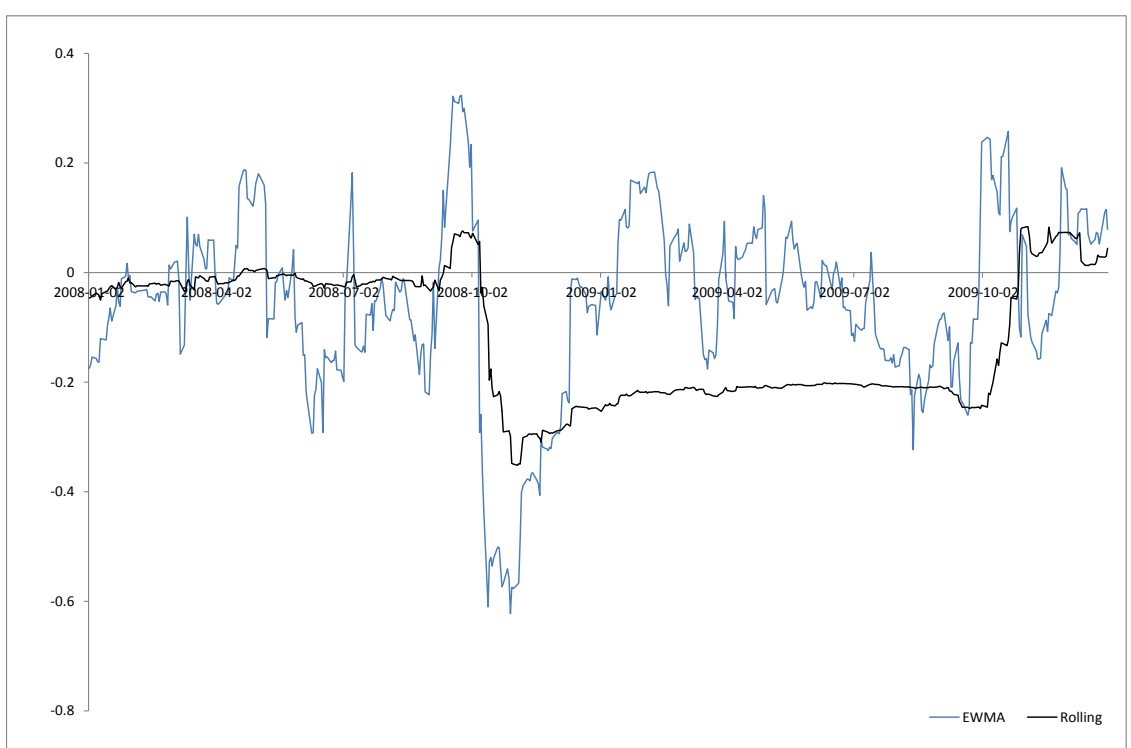

(a) GFC

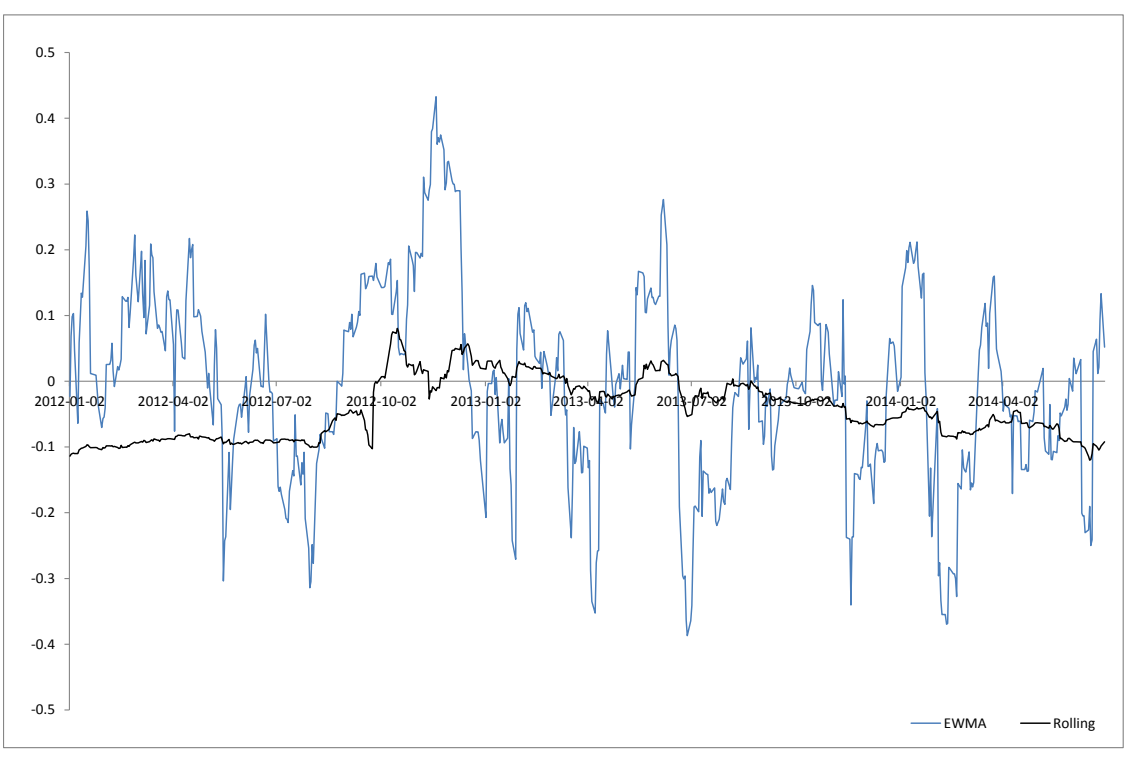

(b) Recent

Figure 3: Correlation dynamics of KOSPI-FX

Note: Plot of rolling linear correlation (252-day moving windows) and EWMA correlation between daily returns of KOSPI and FX.

Figure 3 presents the correlation dynamics of KOSPI-FX. In panel (a), we provide the rolling and EWMA correlations of KOSPI-FX during GFC, and panel (b) present those in Recent period. 
Table 8: Estimation results of DCC models

\begin{tabular}{|c|c|c|c|c|}
\hline & KOSPI-FX & KOSPI-KRX & FX-KRX & KOSPI-FX-KRX \\
\hline$\beta_{1}$ & 0.0000 & $0.0254^{* * *}$ & 0.0202 & $0.0183^{* * *}$ \\
\hline$\beta_{2}$ & $0.9237^{* * *}$ & $0.9676^{* * *}$ & $0.8977^{* * *}$ & $0.9712^{* * *}$ \\
\hline
\end{tabular}

Note: The ${ }^{* * *}$ denotes the statistically significant at $1 \%$ significance level.

The rolling correlation stays around zero from January to October of 2008, and then there is a sharp decreasing and reaches its minimum level at the end of 2008. The negative correlations around -0.2 last almost one year before advent of the increasing momentum at the end of 2009. Moving to EWMA correlation, we see a similar downward spike in October of 2008 as in rolling correlation, and then a gradual increasing momentum. The overall magnitude of rolling and EWMA correlations are -0.117 and -0.06 , respectively. In addition, the rolling correlation of KOSPI-FX in recent period is almost zero with little change, and the EWMA correlations also move along zero level. The strength of rolling correlation is almost zero, except for negative during one year (October $2008 \sim 2009$ ), thus totally negative in Table 3 (the static correlation between KOSPI and FX is -0.1760). Both static and dynamic correlation coefficient are negative, which lead negative Kendall's tau, and thus the parameters of Gumbel and Clayton copulas are unable to estimate. For instance, the relation between the parameter of Gumbel copula and Kendall's tau is $\tau=1-\theta^{-1}$. One get the estimated parameter of Gumbel copula is negative, i.e. outside of its parameter space, if any pair of our interest has a negative Kendall's tau. For similar reason, we cannot find parameter estimate of three-dimensional Gumbel copula.

To examine the correlation dynamics of pairwise returns in Recent, we present the rolling and EWMA correlations in Figure 4. The rolling and EWMA correlations of KOSPI-FX and FX-KRX Bond wander around zero, whereas those of KOSPI-KRX bond show a remarkable increasing after July of 2013, which is lasting almost one year in rolling correlation. We also see that the EWMA correlation of KOSPI-KRX bond increases over times after July of 2013.

To examine three-dimensional correlation dynamics and confirm the robustness of EWMA correlation, we employ DCC of Engle (2002). The parameter estimates of DCC model are reported in Table 8. Similar to GARCH results in Table 3, the sum of $\beta_{1}$ and $\beta_{2}$ is close to one, which indicates a high persistence in correlation between the two series, i.e. a long-run average of the correlation can be removed for a considerably long period by shocks. The EWMA correlation is a special case of DCC. The estimated parameter of DCC models in KOSPI-FX, KOSPI-KRX Bond and KOSPI-FX-KRX Bond are similar as 0.94, thus EWMA correlation shows similar result as in DCC model. We name the correlation of KOSPI-FX-KRX Bond as a system-wide correlation.

Figure 5 shows the conditional correlation dynamics of KOSPI-FX-KRX Bond, obtained from DCC $(1,1)$ model, which provides an insight on dependence structure of Korean financial markets from a systemic perspective. The system-wide correlation shows a clear increasing movement from September 2012 to December 2012. We also see the increasing pattern of pairwise EWMA correlations given in panel (a) of the Figure 4; consequently, we argue that the correlation of KOSPI-FX$\mathrm{KRX}$ Bond is a kind of systemic dependence measure. Interestingly, the system-wide correlation of last one year stays negative values, even the rolling correlation of KOPSI-KRX Bond shows an increasing momentum as in panel (b) of Figure 4.

\section{Conclusion}

This paper investigates the dependence structure of Korean financial markets using copula-GARCH and dynamic conditional correlation models. We examine GJR-GARCH with skewed elliptical distri- 


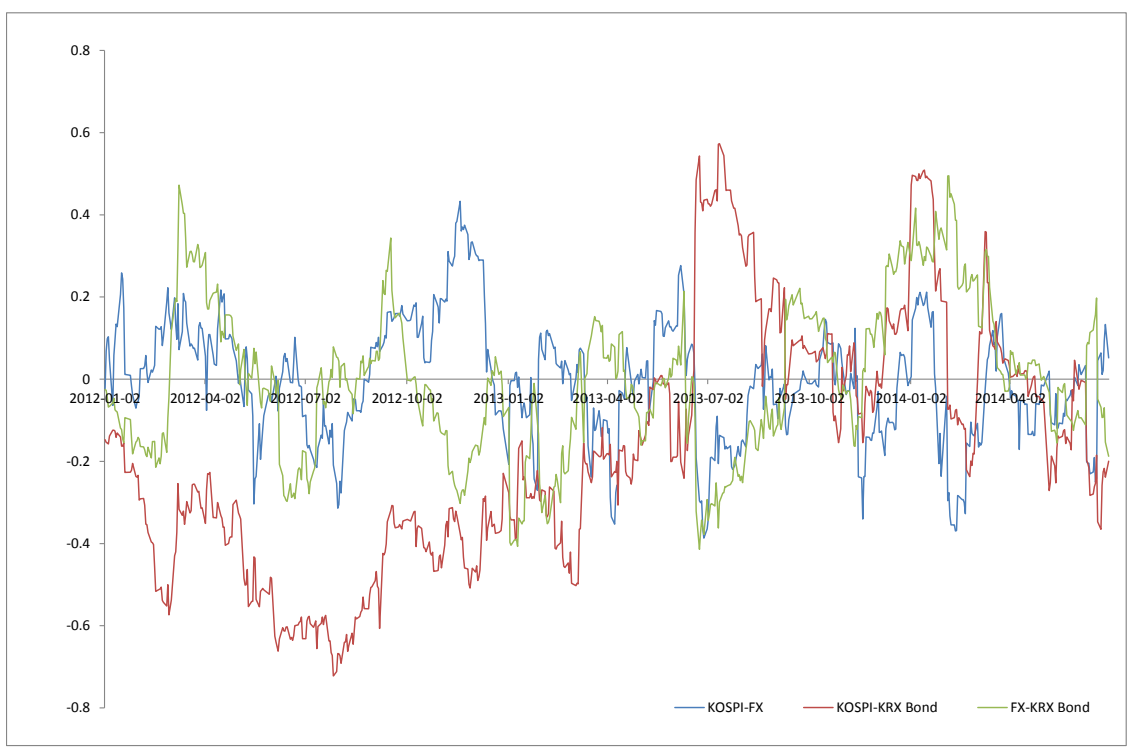

(a) EWMA

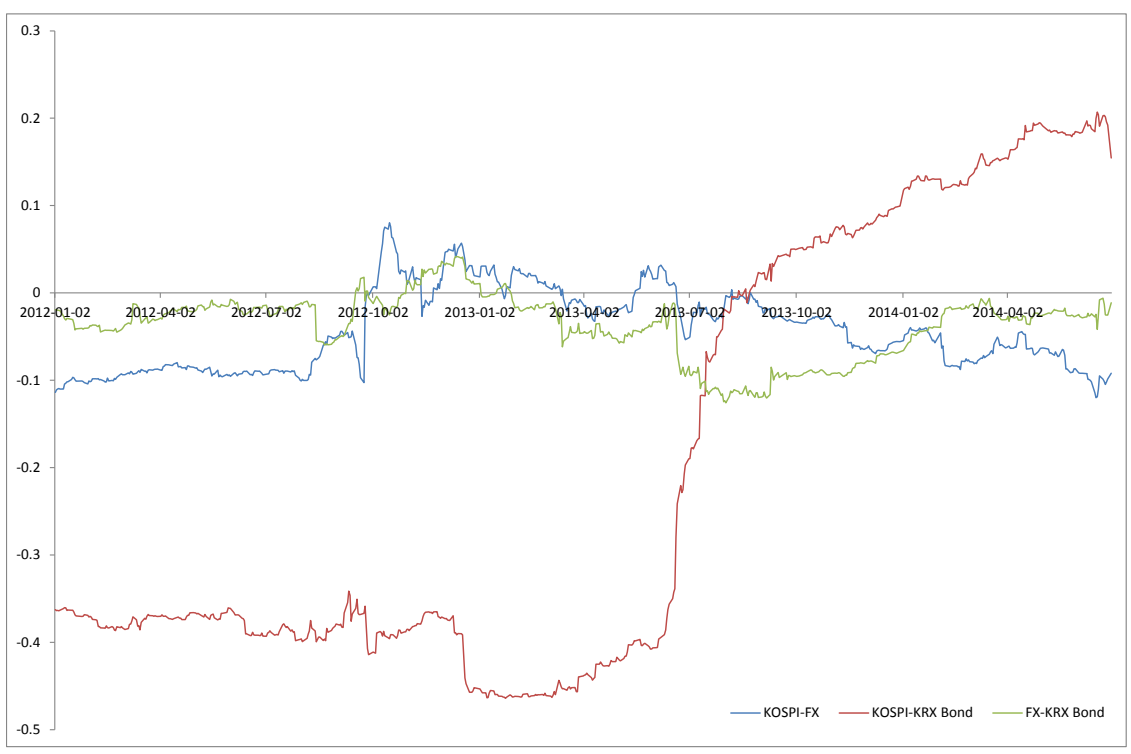

(b) Rolling

Figure 4: Pairwise EWMA and rolling correlation dynamics

Note: Plot of pairwise EWMA correlation and rolling linear correlation (252-day moving windows) between daily returns of KOSPI, FX and KRX Bond for the time period from $2^{\text {nd }}$ January 2012 to $30^{\text {th }}$ June 2014 (616 observations).

butions and four copulas (Gaussian, Student's $t$, Clayton and Gumbel) to model dependence among 


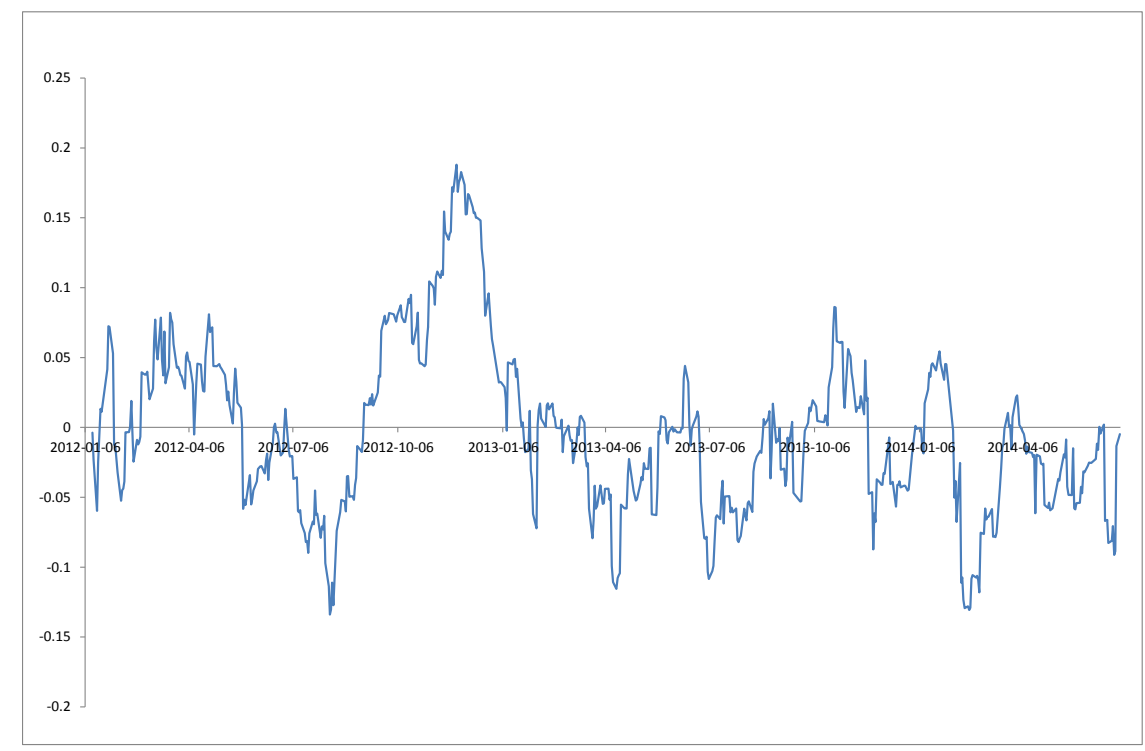

Figure 5: Correlation dynamics of KOSPI-FX-KRX Bond

Note: The dynamic correlation is obtained using $\operatorname{DCC}(1,1)$ with multivariate normal assumption for the time period from $2^{\text {nd }}$ January 2012 to $30^{\text {th }}$ June 2014 (616 observations).

returns incorporating marginal specific characteristics, and then employ DCC model for describing correlation dynamics. We analyze the daily returns of KOSPI, WON/USD and KRX bond index (Gross Price Index), ranged from $2^{\text {nd }}$ May 2006 to $30^{\text {th }}$ June 2014 with 2,063 observations. We find that GJR-GARCH with skew- $t$ distribution is a better candidate during GFC, whereas GJR-GARCH with normal distribution shows good in-sample fit in the recent period, which indicates that there is strong asymmetric effect in Korean market. In addition, our results suggest that there is strong dependence between KOSPI and FX returns during GFC; however, the correlation between stock and bond markets shows a dramatic increase in the recent period. We conclude that the best copula to describe the dependence among Korean markets is $t$ copula, and the estimate of shape parameter is 13 in GFC period, which implies the strong tail dependence among returns, and may lead to simultaneous breakdowns in Korean markets. It is noticeable that the static correlations between pair-wise returns show negligible with negative magnitude; however, dynamic correlations show dramatic movement both up and downward according to market changes. Finally, the system-wide correlation wanders around zero during last two years, which possibly indicates market tranquility in systemic perspective.

\section{Appendix: Dynamic Conditional Correlation Model}

Let $r_{t}$ be the $d$-dimensional vector containing asset returns, which is given as

$$
r_{t}=\mu_{t}+\varepsilon_{t},
$$

where $\mu_{t}$ denotes the conditional mean on the basis on the information up to time $t-1$, which is modeled as constant in this paper. The innovation $\varepsilon_{t}$ is assumed to be a process with $E\left(\varepsilon_{t} \mid F_{t-1}\right)=0$ and $\operatorname{Var}\left(\varepsilon_{t} \mid F_{t-1}\right)=\Sigma_{t}$, where denotes the information set up to time $t-1$. In DCC framework, the 
time varying conditional covariance matrix $\Sigma_{t}$ is decomposed into

$$
\Sigma_{t}=D_{t} \Gamma_{t} D_{t},
$$

where $D_{t}=D_{t}\left(\theta_{1}\right)$ is the $d \times d$ diagonal matrix of time-varying volatilities from one of GARCH models, parameterized by a vector $\theta_{1}$, with $\sigma_{i, t}$ on the $i$ th diagonal. The conditional correlation matrix $\Gamma_{t}=\Gamma_{t}\left(\theta_{1}, \theta_{2}\right)$ contains the time varying correlations between two returns parameterized by two vectors $\theta_{1}$ and $\theta_{2}$.

The correlation matrix $\Gamma_{t}$ of $\operatorname{DCC}(p, q)$ model of Engel (2002) is obtained by modeling a proxy process $Q_{t}$, given by

$$
Q_{t}=\left(1-\sum_{i=1}^{q} a_{i}-\sum_{j=1}^{p} b_{j}\right) \bar{Q}+\sum_{i=1}^{q} a_{i} z_{t-i} z_{t-i}^{\prime}+\sum_{j=1}^{p} b_{j} Q_{t-j}
$$

where $a_{i}$ and $b_{j}$ are non-negative scalars, with the condition that $\sum_{i=1}^{q} a_{i}-\sum_{j=1}^{p} b_{j}<1$ imposed to ensure stationarity and positive definiteness of $Q_{t}$, and $\bar{Q}$ is the sample covariance matrix of standardized errors $z_{t}$, i.e. $\bar{Q}=\frac{1}{T} \sum_{t=1}^{T} z_{t} z_{t}^{\prime}$, where $z_{t}=D_{t}^{-1}\left(r_{t}-\mu_{t}\right)$. The correlation matrix is given as

$$
\Gamma_{t}=\operatorname{diag}\left(Q_{t}^{*}\right)^{-\frac{1}{2}} Q_{t} \operatorname{diag}\left(Q_{t}^{*}\right)^{-\frac{1}{2}},
$$

where $Q_{t}^{*}$ denotes the diagonal matrix of $Q_{t}$.

The estimation of the parameters is done in two steps by quasi-maximum likelihood(QML), assuming that the innovations are Gaussian. The joint log-likelihood of the model can be split into two parts and maximized sequentially. The joint log-likelihood of the model is

$$
L(\theta)=-\frac{1}{2} \sum_{t=1}^{T}\left(d \log (2 \pi)+\log \left|\Sigma_{t}\right|+\varepsilon_{t}^{\prime} \Sigma_{t}^{-1} \varepsilon_{t}\right),
$$

where $\left|\Sigma_{t}\right|$ denotes the determinant of the matrix $\Sigma_{t}$ and $\theta$ is a parameter vector of DCC model. The idea of two-step approach is to separate the correlation parameters from variance parameters. The log-likelihood in (10) can be split into two parts, $L_{v}\left(\theta_{1}\right)$ and $L_{c}\left(\theta_{1}, \theta_{2}\right)$, and maximized sequentially. The log-likelihood in (10) is re-expressed as

$$
L(\theta)=L_{v}\left(\theta_{1}\right)+L_{c}\left(\theta_{1}, \theta_{2}\right),
$$

where

$$
\begin{aligned}
L_{v}\left(\theta_{1}\right) & =-\frac{1}{2} \sum_{t=1}^{T}\left(d \log (2 \pi)+\log \left|D_{t}\right|+\varepsilon_{t}^{\prime} D_{t}^{-1} D_{t}^{-1} \varepsilon_{t}\right), \\
L_{c}\left(\theta_{1}, \theta_{2}\right) & =-\frac{1}{2} \sum_{t=1}^{T}\left(\log \left|\Gamma_{t}\right|+z_{t}^{\prime} \Gamma_{t}^{-1} z_{t}-z_{t}^{\prime} z_{t}\right) .
\end{aligned}
$$

In sequential approach, we estimate the parameters of the volatility model in the first step through to maximize the loglikelihood in formula (A.7). Once $\hat{\theta}_{1}$ is obtained, $\bar{Q}$ is estimated as $1 / T \sum_{t=1}^{T} \hat{z}_{t} \hat{z}_{t}^{\prime}$, where $\hat{z}_{t}=\hat{D}_{t}^{-1}\left(r_{t}-\hat{\mu}_{t}\right)$. In the second step, the parameters of the correlation model are estimated via maximization of loglikelihood $L_{c}\left(\theta_{1}, \theta_{2}\right)$ in formula (A.8). This estimation technique is called as correlation targeting, similar to volatility targeting in univariate GARCH estimation. 


\section{References}

Alouia, R., Aissa, M. S. B. and Nguyen, D. K. (2013). Conditional dependence structure between oil prices and exchange rates: A copula-GARCH approach, Journal of International Money and Finance, 32, 719-738.

Bollerslev, T. (1986). Generalized autoregressive conditional heteroskedasticity, Journal of Econometrics, 31, 300-328.

Dobric, J. and Schmid, F. (2005). Nonparametric estimation of the lower tail dependence in bivariate copulas, Journal of Applied Statistics, 32, 387-407.

Engle, R. F. (2002). Dynamic conditional correlation: A simple class of multivariate GARCH models, Journal of Business and Economic Statistics, 20, 339-350.

Glosten, L. R., Jagannathan, R. and Runkle, D. (1993). On the relation between the expected value and the volatility of the nominal excess return on stocks, Journal of Finance, 48, 1779-1801.

Hillebrand, E. (2005). Neglecting parameter changes in GARCH models, Journal of Econometrics, 129, 121-138.

Huang, J. J., Lee, K. J., Liang, H. and Lina, W. F. (2009). Estimating value at risk of portfolio by conditional copula-GARCH method, Insurance: Mathematics and Economics, 45, 315-324

Joe, H. (1997). Multivariate models and dependence concepts, Monographs in Statistics and Probability 73, Chapman and Hall, London.

Jondeau, E. and Rockinge, M. (2006). The Copula-GARCH model of conditional dependencies: An international stock market application, Journal of International Money and Finance, 25, 827853.

Liu, Y. and Luger, R. (2009). Efficient estimation of copula-GARCH models, Computational Statistics \& Data Analysis, 53, 2284-2297.

Marshal, R. and Zeevi, A. (2002). Beyond correlation: Extreme co-movements between financial assets, Working Paper, Columbia Business.

Mikosch, T. and Starica, C. (2004). Nonstationarities in financial times series, the long range dependence and the IGARCH effects, The Review of Economics and Statistics, 86, 378-390.

Nelsen, R. B. (2006). An Introduction to Copulas, Springer, Second edition, New York.

Pesaran, B. and Pesaran, M. H. (2007). Modelling volatilities and conditional correlations in futures markets with a multivariate $t$ distribution, Cambridge Working Papers in Economics 0734.

RiskMetrics. (1996). RiskMetrics TM Technical Document, Fourth edition, J.P. Morgan/Reuters. 